ISSN 1991-8631

Original Paper

http://indexmedicus.afro.who.int

\title{
Systèmes de production et pratiques à risque en agriculture urbaine : cas du maraîchage dans la ville de Yamoussoukro en Côte d'Ivoire
}

\author{
Bernard Firmin TANO ${ }^{1}$, Kouabenan $\mathrm{ABO}^{2^{*}}$, Ardjouma DEMBELE ${ }^{3}$ et \\ Lassina FONDIO ${ }^{4}$ \\ ${ }^{I}$ Laboratoire National d'Appui au Développement Agricole (LANADA), Laboratoire d'Analyse des Semences \\ (LANASEM). BP 731 Yamoussoukro, Côte d'Ivoire. \\ ${ }^{2}$ Institut National Polytechnique Félix Houphouët Boigny (INP-HB), Département de Formation et de \\ Recherche Agriculture et Ressources Animales, Laboratoire de Phytopathologie et de Biologie Végétale. BP \\ 1313 Yamoussoukro, Côte-d'Ivoire. \\ ${ }^{3}$ Laboratoire National d'Appui au Développement Agricole (LANADA), Laboratoire Central d'Agrochimie et \\ d'Ecotoxicologie (LCAE). 04 BP 612 Abidjan 04, Côte d'Ivoire. \\ ${ }^{4}$ Centre National de Recherche Agronomique (CNRA), Station d'Anguédédou, 01 BP 1740 Abidjan 01, \\ Côte d'Ivoire. \\ *Corresponding author, E-mail: abok65@yahoo.fr
}

\section{RESUME}

La question de l'impact de l'agriculture urbaine sur l'environnement revient, fréquemment, au cœur des débats dans la gestion des villes africaines, comme Yamoussoukro. Les inquiétudes liées à la préservation de l'environnement urbain, eu égard à l'usage de pesticides et à la qualité des eaux utilisées, constituent une préoccupation prise en compte dans le cadre de cette étude. Celle-ci porte, aussi bien sur l'évaluation du risque sur l'environnement consécutif à de mauvaises pratiques agricoles que sur l'évaluation du risque sanitaire consécutif à l'usage des eaux usées. Pour atteindre cet objectif, la méthodologie utilisée a été d'une part, la collecte de données, à travers une enquête et d'autre part, des analyses au laboratoire. Il ressort que le maraîchage urbain à Yamoussoukro est une activité informelle. Les cultures pratiquées sont dominées par la laitue, l'oignon vert et la carotte. Le recours aux intrants, notamment les pesticides, est très répandu. Les dispositifs règlementaires sur la distribution des produits agrochimiques ne sont pas appliqués. Ainsi, 74\% des pesticides recensés ne sont pas homologués pour les cultures maraîchères, $12 \%$ sont très toxiques (classe $\mathrm{Ib}$ ) et 57\% nocifs (classe II). Les risques encourus sont accentués par un traitement chimique qui n'est pas suffisamment maîtrisé. Les analyses physico-chimiques et microbiologiques ont mis en évidence, d'une part, des eaux d'irrigation aux caractéristiques très hétérogènes et d'autre part, une grande diversité microbienne et une contamination de ces eaux, suggérant des risques sanitaires avérés.

(C) 2011 International Formulae Group. All rights reserved.

Mots clés : cultures maraîchères, environnement, risque sanitaire, pesticides, eaux.

\section{INTRODUCTION}

L'agriculture urbaine à Yamoussoukro, capitale politique de la Côte d'Ivoire, est essentiellement dominée par les cultures maraîchères (Adama et Michel, 2004 ;
ANADER, 2004). Les productions permettent d'approvisionner le marché local de la ville, mais aussi ceux des villes environnantes et d'Abidjan plus au Sud (Abakou et Brou, 2003). Toutefois, ce résultat, comme dans 
plusieurs villes africaines, est le fait d'un système de production en milieu urbain soumis à plusieurs contraintes (Obuodi et al., 2006 ; N'guegang, 2008). Elle est exigeante à propos de la gestion de la fertilité du sol et de l'approvisionnement en eau afin d'assurer une production horticole intensive toute l'année. En effet, à l'image de nombreuses cités, en milieu tropical, l'emploi permanent d'engrais, de pesticides et l'usage des eaux usées, sont des pratiques largement répandues dans le maraîchage urbain à Yamoussoukro (Bendé, 2003 ; Amoah et al., 2006). Elles peuvent constituer une source de pollution et une menace pour la santé des populations de la ville et celle des consommateurs des produits récoltés (Amoah et al., 2006). Aussi, l'utilisation de diverses sources d'eau d'irrigation dont les eaux usées de la ville est un fait accompli. Les risques sanitaires liés à la contamination des cultures par des organismes pathogènes sont élevés. Ainsi, il s'est agi, dans le cadre de cette étude, d'évaluer les facteurs de risques consécutifs à l'usage des intrants et des eaux usées dans le système de production intensif des cultures maraîchères dans la ville de Yamoussoukro. Pour atteindre cet objectif, une démarche méthodologique, en deux points a été adoptée : la collecte de données de terrain, au moyen d'une enquête pour cerner et caractériser l'environnement de production des cultures marâichères urbaines, des analyses physico-chimiques et microbiologiques en laboratoire, pour évaluer la qualité des eaux d'arrosage.

\section{MATERIEL ET METHODES Enquête}

L'enquête a concerné deux groupes de populations cibles: les maraîchers et les distributeurs de produits phytosanitaires. Chaque groupe cible a été soumis à un questionnaire particulier. Un échantillon de 66 maraîchers, sur un total de 220 recensés en 2004 par l'Agence Nationale d'Appui au Développement Rural (ANADER), a été interrogé. Au niveau des distributeurs, sept points de vente répartis sur les deux marchés de la ville et un magasin spécialisé ont été visités. Les données collectées ont été dépouillées aussi bien manuellement qu'à l'aide du tableur Excel.

\section{Etude de la qualité des eaux d'arrosage Echantillonnage et description des sites de prélèvement}

Des prélèvements d'eau ont été effectués sur 8 sites de production en fonction de la nature de ces eaux et de la densité des maraîchers. Deux types d'analyses ont été effectuées : (i) des analyses physicochimiques au laboratoire de chimie du Département Génie chimique et Agroalimentaire de l'INP-HB à Yamoussoukro, selon les méthodes normalisées AFNOR; (ii) des analyses bactériologiques au Laboratoire d'hygiène alimentaire du Laboratoire National d'Appui au Développement Agricole (LANADA) à Abidjan, par la méthode dite de filtration sur membrane. Trois prélèvements espacés d'environ deux semaines ont été effectués. Ces prélèvements étaient effectués le matin, entre 8 et 10 heures. Les 8 points de prélèvement sont: P1 (lac pollué des jardins de Guiglo), P2 (puits non protégé au bord du lac de Guiglo), P3 (eaux d'assainissement derrière les bureaux de la poste), $\mathrm{P} 4$ (puits non protégé derrière les bureaux de la Compagnie Ivoirienne d'Electricité), P5 (Eaux stagnantes dans un bas-fond du quartier Zaherbougou), P6 (bas-fond alimenté par des eaux usées derrière la Fondation Internationale Félix Houphouët Boigny pour la Recherche de la Paix), P7 (lac non pollué situé derrière le camp de la Garde Républicaine) et P8 (bac d'arrosage du site d'implantation de la FAO). Les zones de production et les points de prélèvement sont indiqués dans la Figure 1.

Critères d'appréciation de la qualité des eaux

Pour apprécier la qualité des eaux étudiées, il est utile de disposer de critères de comparaison. Les classes de qualité utilisées sont celles employées en France pour les eaux de rivières (Delarras et Trébaol, 2003; Rejsek, 2002). Ces classes sont 1A, 1B, 2, 3 et hors classe, décrivant l'eau la plus excellente à la moins bonne dite hors classe. Pour déterminer l'appartenance d'une eau à une classe donnée, les valeurs d'un certain nombre de paramètres sont étudiées. Ces valeurs sont indiquées dans le Tableau 1. 


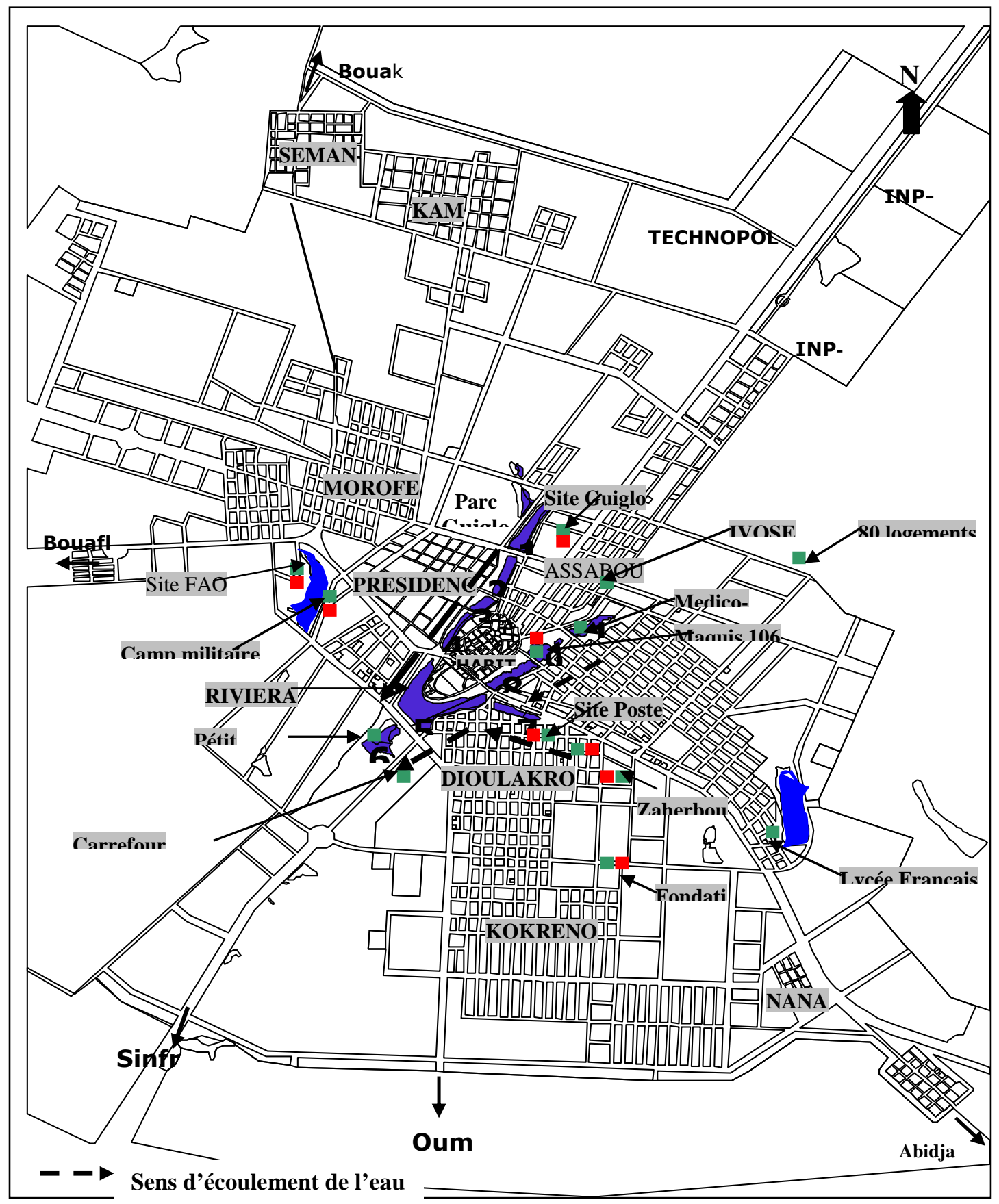

Légende :

Zones de productions visitées
Points de prélèvements
Lacs artificiels

Echelle : 1/100 000

Figure 1 : Localisation des zones de production et des points de prélèvement. 
Tableau 1 : Classes d'analyse et normes des paramètres mesurés.

\begin{tabular}{|c|c|c|c|c|c|c|}
\hline Paramètres & Unités & $\begin{array}{l}\text { Classe } \\
1 \text { A }\end{array}$ & $\begin{array}{l}\text { Classe } \\
1 \mathrm{~B}\end{array}$ & $\begin{array}{l}\text { Classe } \\
2\end{array}$ & $\begin{array}{l}\text { Classe } \\
3\end{array}$ & Hors classe \\
\hline Azote ammoniacal & $\mathrm{mg} \mathrm{NH} 4+/ \mathrm{L}$ & $<0,1$ & 0,1 à 0,5 & 0,5 à 2 & 2 à 8 & $>8$ \\
\hline Azote kjeldhal & $\mathrm{mg} \mathrm{N} / \mathrm{L}$ & $<1$ & 1 à 2 & 2 à 3 & $>3$ & $>3$ \\
\hline Chlorures & $\mathrm{mg} \mathrm{Cl}-/ \mathrm{L}$ & $<100$ & 100 à 200 & 200 à 400 & 400 à 1000 & $>1000$ \\
\hline Conductivité & $\mathrm{US} / \mathrm{cm}$ & $<400<$ & 400 à 750 & 750 à 1500 & 1500 à 3000 & $>3000$ \\
\hline Nitrates & mg NO3-/L & $<5$ & 5 à 25 & 25 à 50 & 50 à 100 & $>100$ \\
\hline Orthophosphates & mg PO43-/L & $<, 2$ & 0,2 à 0,5 & 0,5 à 1 & 1 à 5 & $>5$ \\
\hline Phosphore total & $\mathrm{mg} \mathrm{P} / \mathrm{L}$ & $<0,1$ & 0,1 à 0,25 & 0,25 à 0,5 & 0,5 à 2,5 & $>2,5$ \\
\hline $\mathrm{DBO}_{5}$ & $\mathrm{mg} \mathrm{O} 2 / \mathrm{L}$ & $<3$ & 3 à 5 & 5 à 10 & 10 à 25 & $>25$ \\
\hline Indice permanganate & $\mathrm{mg} \mathrm{O} 2 / \mathrm{L}$ & $<3$ & 3 à 5 & 5 à 8 & $>8$ & $>8$ \\
\hline MES & $\mathrm{mg} / \mathrm{L}$ & $<30$ & $<30$ & $<30$ & 30 à 70 & $>70$ \\
\hline Oxygène dissous & $\%$ saturation & $>90$ & 70 à 90 & 50 à 70 & $<50$ & $<50$ \\
\hline Oxygène dissous & $\mathrm{mg} \mathrm{I} 2 / \mathrm{L}$ & $>7$ & 5 à 7 & 3 à 5 & 0 à 3 & 0 à 3 \\
\hline $\mathrm{pH}$ & -- & 6,5 à 8,5 & 6,5 à 8,5 & 6 à 6,5 ou 8,5 à 9 & 5,5 à 6 ou 9 à 9,5 & $<5,5$ ou $>9,5$ \\
\hline Coliformes totaux & $\mathrm{U} / 100 \mathrm{ml}$ & $<50$ & 50 à 5000 & 5000 à 50000 & $>50000$ & $>50000$ \\
\hline Escherichia coli & $\mathrm{U} / 100 \mathrm{ml}$ & $<20$ & 20 à 2000 & 2000 à 20000 & $>20000$ & $>20000$ \\
\hline Enterocoques fécaux & $\mathrm{U} / 100 \mathrm{ml}$ & $<20$ & 20 à 1000 & 1000 à 10000 & $>10000$ & $>10000$ \\
\hline
\end{tabular}




\section{RESULTATS}

Caractéristiques des producteurs et pratiques culturales

Les producteurs

Le maraîchage urbain à Yamoussoukro est exercé majoritairement par les hommes avec $88 \%$ des producteurs. Il est tenu, par des non nationaux $(71 \%)$, essentiellement des burkinabés et des maliens. C'est une activité ouverte à tous les âges. Les producteurs sont en majorité illettrés (81\%), Le maraîchage est une activité informelle à Yamoussoukro, caractérisée par une quasi absence de regroupement des producteurs qui entretiennent des exploitations individuelles (91\%).

\section{Les sites de production}

Un total de 13 grands sites de production ont été répertoriés à l'intérieur et aux extrémités de la ville. Ils se retrouvent, préférentiellement, autour des lacs artificiels disséminés dans la ville, avec $54 \%$ des sites ; $31 \%$ des sites se situent dans des bas-fonds de collecte d'eaux usées et $15 \%$ dans les basfonds humides ou aménagés à la périphérie de la ville. Les installations spontanées sans autorisation préalable ou «squatting» représentent $89 \%$ du mode d'accès à la terre.

La taille des exploitations et types de cultures

Les exploitations sont de tailles très variées, sur un même site, mais aussi d'un site à l'autre. Les domaines d'exploitations sont relativement petits. La majorité des exploitations (69\%) ont une superficie comprise entre $100 \mathrm{~m}^{2}$ et $1000 \mathrm{~m}^{2}$. Le marâichage urbain à Yamoussoukro se caractérise par une polyculture où plusieurs cultures sont pratiquées par chaque producteur sur différentes parcelles. Plus d'une quinzaine de spéculations sont rencontrées. Pour les plus cultivées, les taux d'adoption par les producteurs sont par ordre décroissant : 79\%, $76 \%$ et $53 \%$, respectivement pour la laitue, l'oignon vert et la carotte (Figure 2).

\section{Les intrants}

Deux groupes de distributeurs se partagent le marché de la vente des intrants à Yamoussoukro: d'une part ceux qui sont agréés pour la vente des produits phytosanitaires; ils ne sont que deux, un grossiste et un magasin spécialisé qui est une représentation de la firme STEPC basée à Abidjan, d'autre part, nous avons les revendeurs qui n'ont pas d'agrément. Ils sont les plus nombreux. Hormis les produits liquides, tous les autres produits sont reconditionnés dans des emballages sans étiquettes pour être revendus en détail. Leurs sources d'approvisionnement sont les gros magasins et quelquefois, des firmes à Abidjan. Le niveau d'instruction de ces distributeurs est faible (cours moyen). Les fumures organiques, essentiellement les fientes de poulet, disponibles en quantité sur les sites, sont vendues par les fermes avicoles environnantes. L'ANADER, structure étatique d'encadrement, intervient, en conseillant les producteurs, pour l'approvisionnement en intrants, notamment les semences sélectionnées ou améliorées d'aubergine et d'oignon.

La fertilisation du sol se fait par l'apport d'engrais organiques et minéraux. La Figure 3 présente le niveau d'adoption pour chaque type d'engrais. L'urée, les NPKs et les fientes de poulet sont les fertilisants les plus utilisés par les maraîchers (respectivement $94 \%, 88 \%$, et $86 \%$ ). Les doses d'application, très hétérogènes, varient d'un producteur à l'autre. Elles peuvent être regroupées comme suit:

- NPKs : $1 \mathrm{~kg}$ pour 30 à $112,5 \mathrm{~m}^{2}$ soit 89

à $333 \mathrm{~kg}$ par hectare ;

- urée : $1 \mathrm{~kg}$ pour 40 à $112.5 \mathrm{~m}^{2}$ soit 89 à

$250 \mathrm{~kg}$ par hectare ;

- fientes de poulets : 40 à $50 \mathrm{~kg}$ pour 30 à

$90 \mathrm{~m}^{2}$ soit 4444 à $16666 \mathrm{~kg}$ par hectare.

Afin de faire face à la forte pression parasitaire, les maraîchers utilisent une gamme variée de produits phytosanitaires 
(Tableau 2). L'enquête auprès des maraîchers de Yamoussoukro a permis de répertorier 27 noms commerciaux de pesticides et 16 matières actives dominées par les pyréthrinoïdes $(27 \%)$ et les organochlorés (19\%). La classification selon les cibles laisse percevoir une nette dominance des insecticides (42\%), suivis des fongicides (27\%) puis les produits à large spectre d'action que sont les insecticides-acaricides (19\%) et les insecticides-nématicides 12\% (Figure 4). $\mathrm{Au}$ niveau des formulations, les concentrés émulsifiables (EC), avec 54\%, sont les plus utilisés, suivis des poudres mouillables (WP), pour $17 \%$. Concernant la spécificité des pesticides utilisés, les produits homologués pour le cotonnier représentent $41 \%$; les produits homologués pour les cultures maraîchères représentent $26 \% ; 14 \%$ sont spécifiques au binôme ananas/banane et $19 \%$ sont spécifiques à des cultures diverses (cacao, palmiers à huile, etc.). Le traitement est soit réalisé à l'aide d'un pulvérisateur à pression entretenue (59\% des maraîchers), soit à l'aide d'un arrosoir (41\% des maraîchers). Les doses pratiquées d'un producteur à l'autre sont très hétérogènes. Le Tableau 3 indique les doses d'application des principaux pesticides que nous avons recensé. Le traitement des cultures est pratiqué par tous les maraîchers. Il se caractérise par la diversité et surtout par les associations plus ou moins complexes des pesticides. Parmi les pratiques qui se démarquent, on peut noter l'utilisation d'un seul insecticide $(21 \%)$ dont la part de Decis représente (17\%). L'association insecticide - fongicide (28\%) dominée par l'association Decis / Calliman (24\%). La répartition des producteurs, en fonction du délai d'emploi avant récolte, montre que 56\% de ceux-ci programment leurs récoltes dans les 3 jours qui suivent le dernier traitement; $32 \%$ récoltent une semaine après et seulement
$12 \%$ entre $10-15$ jours après le dernier traitement.

Seuls 3\% des producteurs ont une bonne connaissance des pictogrammes de danger et $87 \%$ ne portent aucun équipement de protection (gants, masques). Soixante dix $(70 \%)$ des producteurs ne voient pas en l'usage des pesticides une source de danger pour l'environnement et $76 \%$ rejettent les emballages vides dans la nature.

\section{Caractéristiques physico-chimiques et biologiques des eaux d'arrosage}

Pour chaque paramètre, les moyennes des valeurs mesurées sont présentées dans le Tableau 4. Selon la grille de qualité rappelée dans le Tableau 1, tous les points d'irrigation analysés sont aptes à l'irrigation par rapport aux paramètres suivants : température, $\mathrm{pH}$, l'indice permanganate, DBO5, nitrate et conductivité. Par contre, le taux d'oxygène du point P2, de 2,91 $\mathrm{mg} / \mathrm{L}$, est source de pollution excessive par rapport à ce paramètre. De même, les valeurs mesurées des MES (Matières en suspension) des points d'irrigations $\mathrm{P} 1, \mathrm{P} 2$ et $\mathrm{P} 6$, respectivement 91, 93 et $88 \mathrm{mg} / \mathrm{L}$, sont sources de pollution excessive et ne sont donc pas aptes à l'irrigation. En dehors de la température et du $\mathrm{pH}$, tous les autres paramètres sont très hétérogènes, notamment le nitrate qui a un coefficient de variation (CV) de $80 \%$. Les résultats microbiologiques (Tableau 5) montrent un large spectre de contaminations dans les différents points d'eau d'irrigation. Les trois paramètres microbiologiques que sont les coliformes totaux, E. Coli, et streptocoques fécaux sont présents dans tous les points d'eau d'irrigation. Le point P3 est positif à tous les paramètres choisis ; il est, du reste, le seul qui est contaminé par des salmonelles. 
B. F. TANO et al. / Int. J. Biol. Chem. Sci. 5(6): 2317-2329, 2011

Tableau 2 : Produits phytosanitaires utilisés par les maraîchers enquêtés.

\begin{tabular}{|c|c|c|c|c|c|c|c|}
\hline Nom commercial & Matières actives & Concentrations & Familles & Classe toxico. & Formulations & H.C.M & Spécificités \\
\hline \multicolumn{8}{|c|}{ INSECTICIDES } \\
\hline BIOBIT & & & Produit biologique & & & Oui. & \\
\hline Cypercal 50 EC & Cyperméthrine & $50 \mathrm{~g} / 1$ & Pyréthrinoïde & III & $\mathrm{EC}$ & Oui. & \\
\hline Cypercal P 186 EC & $\begin{array}{l}\text { Cyperméthrine+ } \\
\text { profénofos }\end{array}$ & $\begin{array}{l}36 \mathrm{~g} / \mathrm{l}+ \\
150 \mathrm{~g} / \mathrm{l}\end{array}$ & $\begin{array}{c}\text { Pyréthrinoïde } \\
\text { Organophosphoré }\end{array}$ & III & $\mathrm{EC}$ & Non & Coton \\
\hline Decis $12 \mathrm{EC}$ & Deltaméthrine & $12,5 \mathrm{~g} / \mathrm{l}$ & Pyréthrinoïde & II & $\mathrm{EC}$ & Oui.. & \\
\hline Dursban $5 \mathrm{G}$ & Chlorpyriphos-éthyl & $50 \mathrm{~g} / 1$ & Pyréthrinoïde & III & GR & Non. & Coton \\
\hline Dursban 150/18 EC & $\begin{array}{l}\text { Cyfluthrine+ } \\
\text { Chlorpyriphos-éthyl }\end{array}$ & $\begin{array}{c}18 \mathrm{~g} / 1+ \\
150 \mathrm{~g} / 1\end{array}$ & $\begin{array}{l}\text { Organophosphoré } \\
\text { Pyréthrinoïde }\end{array}$ & $\mathrm{I} b$ & $\mathrm{EC}$ & Non & Coton \\
\hline Karaté 5EC & Lambdacyhalothrine & $37,5 \mathrm{~g} / \mathrm{kg}$ & Pyréthrinoïde & II & $\mathrm{EC}$ & Non & Coton \\
\hline KART 500 SP & Cartap & $500 \mathrm{~g} / \mathrm{kg}$ & Carbamate & I & SP & Non & Palmier à huile \\
\hline Marshall 35 DS & Carbosuran & $350 \mathrm{~g} / 1$ & Carbamate & II & DS & Non & Coton \\
\hline Orthène 75 SP & Acephate & $750 \mathrm{~g} / \mathrm{kg}$ & Organophosphoré & III & SP & Oui. & \\
\hline Phaser Ultra CAPS & Endosulfan & $330 \mathrm{~g} / 1$ & Organochloré & II & $\mathrm{CS}$ & Non & Coton \\
\hline Phaser 350 EC & Endosulfan & $350 \mathrm{~g} / 1$ & Organochloré & & $\mathrm{EC}$ & Non & Coton \\
\hline Polytrine C 186 EC & $\begin{array}{l}\text { Cyperméthrine+ } \\
\text { Profenofos }\end{array}$ & $\begin{array}{l}36 \mathrm{~g} / \mathrm{l}+ \\
150 \mathrm{~g} / \mathrm{l}\end{array}$ & $\begin{array}{c}\text { Pyréthrinoïde } \\
\text { Organophosphoré }\end{array}$ & II & $\mathrm{EC}$ & Non & Coton \\
\hline Regent 50 SC & Fipronil & $50 \mathrm{~g} / 1$ & Phénypyrazole & II & $\mathrm{SC}$ & Non. & Bananier \\
\hline \multicolumn{8}{|c|}{ INSECTICIDES-ACARICIDES } \\
\hline Caïman $350 \mathrm{EC}$ & Endosulfan & $350 \mathrm{~g} / \mathrm{l}$ & Organochloré & & $\mathrm{EC}$ & Non & Coton \\
\hline Endocoton $375 \mathrm{EC}$ & Endosulfan & $375 \mathrm{~g} / 1$ & Organochloré & I & $\mathrm{EC}$ & Non & Coton \\
\hline Phaser 375 EC & Endosulfan & $375 \mathrm{~g} / \mathrm{l}$ & Organochloré & $\mathrm{Ib}$ & $\mathrm{EC}$ & Non & Coton \\
\hline \multicolumn{8}{|c|}{ NEMATICIDES-INSECTICIDES } \\
\hline Furadan $5 \mathrm{G}$ & Carbofuran & $5 \%$ & Carbamate & $\mathrm{I}$ & GR & Non & Ananas/banane \\
\hline Marshall 250 EC & Carbosulfan & $250 \mathrm{~g} / 1$ & Carbamate & II & $\mathrm{EC}$ & Non & Ananas/banane \\
\hline
\end{tabular}


B. F. TANO et al. / Int. J. Biol. Chem. Sci. 5(6): 2317-2329, 2011

\begin{tabular}{|c|c|c|c|c|c|c|c|}
\hline \multicolumn{8}{|c|}{ FONGICIDES } \\
\hline Alliette $80 \mathrm{WP}$ & Fosetyl-Al & $800 \mathrm{~g} / \mathrm{kg}$ & Monoéthyl-phosphite & III & WP & Non & Ananas/banane \\
\hline BBS & Sulphate cuivre & $2 \%$ & & & & Non & Cacao \\
\hline Callicuivre & Oxychlorure de cuivre & $50 \%$ & & III & PM & Oui. & \\
\hline Calliman 80 & Manèbe & $800 \mathrm{~g} / \mathrm{kg}$ & Dithiocarbamate & III & WP & Oui. & \\
\hline Trimangol $80 \mathrm{Pm}$ & Manèbe & $800 \mathrm{~g} / \mathrm{kg}$ & Dithiocarbamate & III & WP & Oui. & \\
\hline Manate 80 & Manèbe & $800 \mathrm{~g} / \mathrm{kg}$ & Dithiocarbamate & III & WP & Oui. & \\
\hline
\end{tabular}

H.C.M : Homologué pour les Cultures Maraîchères

Tableau 3 : Doses des pesticides utilisées et types de matériel d'application.

\begin{tabular}{|c|c|c|}
\hline \multirow{2}{*}{ Produits } & \multicolumn{2}{|c|}{ Doses } \\
\hline & Avec un pulvérisateur & Avec un arrosoir \\
\hline & $20-40 \mathrm{ml} / 15-40 \mathrm{l}$ & $20 \mathrm{ml} / 40-601 \mathrm{~d}$ 'eau $/ 48-72 \mathrm{~m}^{2}$ \\
\hline Decis & d'eau / 240-1200 m² & $40 \mathrm{ml} / 80-1201$ d'eau / 60-90 m² \\
\hline $\begin{array}{l}\text { Endocoton } \\
\text { Cypercal } \\
\text { Phaser } \\
\text { Dursban }\end{array}$ & $40 \mathrm{ml} / 15-201$ d'eau / 250-500 m² & $40 \mathrm{ml} / 80-1201$ d'eau / 60-90 m² \\
\hline Kart 500 SP & $15 \mathrm{~g} / 161$ d'eau $/ 500 \mathrm{~m}^{2}$ & - \\
\hline $\begin{array}{l}\text { Calliman } 80 \\
\text { Alliette } 80 \mathrm{WP} \\
\text { Manate } \\
\text { Callicuivre }\end{array}$ & $50 \mathrm{~g} / 15-301$ d'eau / 250-400 $\mathrm{m}^{2}$ & $50 \mathrm{~g} / 200-3201 / 150-205 \mathrm{~m}^{2}$ \\
\hline
\end{tabular}


B. F. TANO et al. /Int. J. Biol. Chem. Sci. 5(6): 2317-2329, 2011

Tableau 4 : Caractéristiques physico-chimiques des points d'eau d'irrigation.

\begin{tabular}{lcccccccccc}
\hline \multirow{2}{*}{ Paramètres } & \multicolumn{7}{c}{ Points d'eaux d'irrigation } & CV (\%) \\
\cline { 2 - 7 } & P1 & P2 & P3 & P4 & P5 & P6 & P7 & P8 & $(*)$ \\
\hline $\mathrm{T}^{\circ}$ & 27,65 & 26,8 & 26,6 & 27,45 & 26,6 & 26,3 & 27,85 & 27,2 & 2 \\
PH & 7,3 & 6,96 & 7,1 & 7,1 & 7,14 & 6,81 & 7,38 & 7,705 & 4 \\
Oxygène dissous & 3,065 & 1,515 & 3,576 & 4,965 & 4,51 & 3,59 & 4,88 & 5,97 & 32 \\
I P & 3,75 & 7,61 & 4,165 & 4,085 & 1,78 & 1,9 & 4,28 & 3,03 & 45 \\
D B O & 5,725 & 10,85 & 4,98 & 5,975 & 5,5 & 3,85 & 4,79 & 4,25 & 36 \\
M E S & 91 & 93 & 15,5 & 22 & 37,5 & 88 & 40 & 17,5 & 64 \\
Nitrate & 0,01 & 0,09 & 0,05 & 0,02 & 0,025 & 0,01 & 0,025 & 0,02 & 80 \\
Conductivité & 298,4 & 366 & 631,5 & 490 & 446 & 209,3 & 297,5 & 293,9 & 34 \\
\hline
\end{tabular}

$(*)$ : Coefficient de Variation

Tableau 5 : Résultats des analyses microbiologiques.

\begin{tabular}{|c|c|c|c|c|c|c|c|c|}
\hline \multirow{2}{*}{ Paramètres } & \multicolumn{8}{|c|}{ Points d'eaux d'irrigation } \\
\hline & P1 & $\mathbf{P 2}$ & $\mathbf{P 3}$ & P4 & P5 & P6 & P7 & P8 \\
\hline Aérobies mésophiles totales & + & + & + & + & + & + & + & + \\
\hline Coliformes totaux/g & + & + & + & + & + & + & + & + \\
\hline Coliformes fécaux/g & + & + & + & + & + & + & + & + \\
\hline Eschericha coli & + & + & + & + & + & + & + & + \\
\hline Staphylocoques dorés/g & - & + & + & + & - & + & + & + \\
\hline Anaérobies sulfito-réducteurs $46^{\circ} \mathrm{C} / \mathrm{g}$ & - & + & + & - & - & + & - & + \\
\hline Streptocoques fécaux/g & + & + & + & + & + & + & + & + \\
\hline Salmonelles/25g & - & - & + & - & - & - & - & - \\
\hline
\end{tabular}




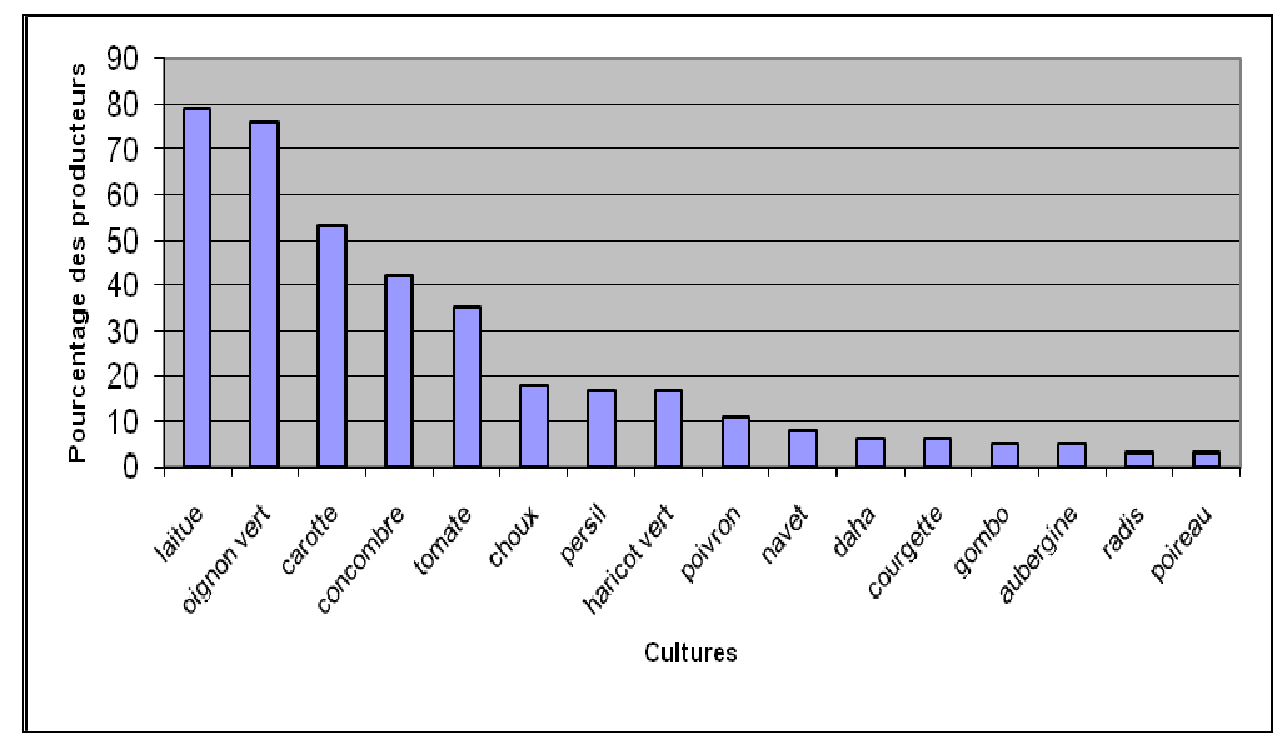

Figure 2 : Répartition des maraîchers en fonction du niveau de choix des cultures.

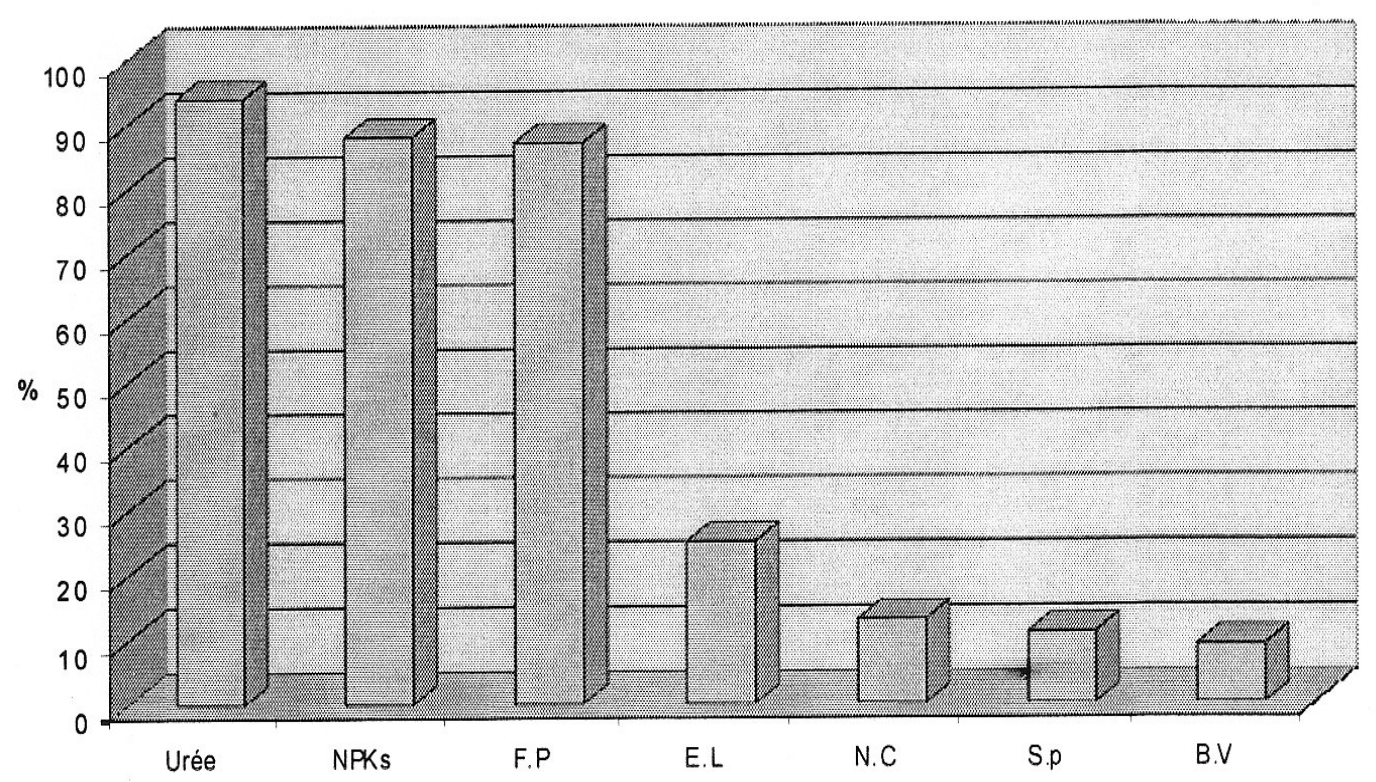

Figure 3 : Niveau d'adoption des différents fertilisants par les producteurs. F.P : fiente de poulet ; E.L : engrais liquides ; N.C : nitrate de calcium ; S.P : sulfate de potassium ; B.V : bouse de vache. 


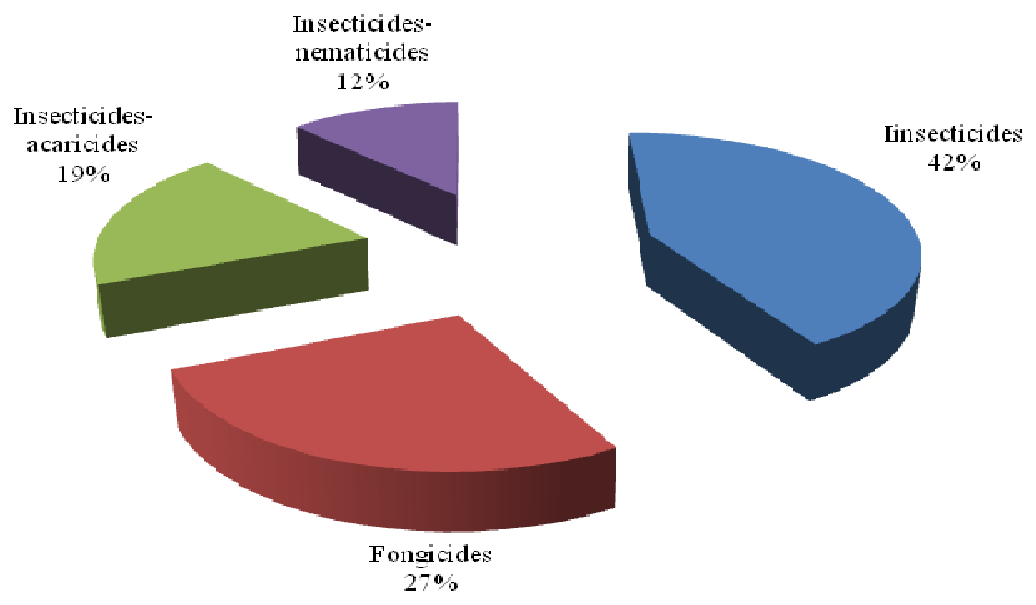

Figure 4 : Répartition des pesticides selon les cibles.

\section{DISCUSSION}

Cette étude montre d'abord une diversité de cultures pratiquées par les producteurs, dominées, à l'occasion, par la laitue, l'oignon vert et la carotte. C'est un système de production caractérisé, entre autres, par la pluralité des cultures adoptées. Cette situation n'est guère différente de celles observées dans d'autres cités africaines (Maïga et al., 2002; Obuodi et al., 2006 ; Fondio et al., 2011). Toutefois, à l'issue de l'enquête sur le comportement des producteurs, il apparaît une méconnaissance par les maraîchers, de l'impact de certaines pratiques sur l'environnement et la santé des potentiels consommateurs. Cela se justifie, entre autres, par la faible part des produits homologués utilisés pour la protection des cultures maraîchères (26\%). En 2002, elle représentait $40 \%$ (Diaby, 2002) et seuls les produits cotonniers étaient étrangers aux cultures maraîchères. La présence de produits non homologués pour ces cultures est un risque important pour la filière horticole ellemême (risques de toxicité de tout genre), pour l'environnement (Dümler et al., 1993 ; Amoah et al., 2006). Le non respect des délais d'application avant récolte, couplé à la toxicité excessive de certains produits $(56 \%$ appartenant à la classe II et $12 \%$ à la classe I) (Kouablé, 2002) expose à des risques de dépassement des limites maximales de résidus (LMR) et d'intoxication du consommateur surtout que les principales cultures pratiquées par les producteurs sont la laitue et l'oignon vert; primeurs sensibles ; souvent consommés frais. Par ailleurs, les maraîchers procèdent à des mélanges dont ils ne maîtrisent ni le dosage, ni la rémanence, encore moins les propriétés physico-chimiques. Les mélanges des matières actives ainsi que les adjuvants des formulations pourraient avoir des effets additifs, de synergie, d'antagonisme. Ce qui se traduit par des risques de phytotoxicité, de rémanence accrue dans l'environnement, mais aussi des risques d'inefficacité du traitement et de détérioration du matériel (Tron et al., 2001; Thiam, 2007). Les concentrations excessives des matières en suspension au niveau de certains points d'irrigation, la diversité et la forte présence des bactéries dans les différents points d'eau, dénotent de la vulnérabilité de ceux-ci face aux contaminations extérieures (Tellier, 2006). Cette situation s'expliquerait par la topographie de certains sites de production. En effet, les sources d'eau d'irrigation en bas de pente sont des collecteurs des eaux de ruissellement de qualité variée, certaines plus sujettes à des risques importants de pollution (pollution d'origine domestique, pollution d'origine agricole, notamment par les 
déjections animales utilisées comme fertilisants). Le point d'irrigation P3 (eaux d'assainissement derrière les bureaux de la poste), plus que les autres, inquiète par la présence de salmonelles. Cela se justifie aisément quand on sait qu'il est principalement alimenté par des eaux des caniveaux de la ville. Cette situation a été démontrée par d'autres études, en l'occurrence celles de Ntow (2001) et de Amoah et al. (2006) au Ghana.

\section{Conclusion}

L'étude des risques environnementaux consécutifs au maraîchage urbain met en évidence les mauvaises pratiques maraîchères. En effet, le maraîchage urbain à Yamoussoukro est une activité non organisée, pratiquée essentiellement par des producteurs illettrés livrés à eux-mêmes dans la ville. Les sites de production sont situés à proximité des sources d'eau d'irrigation que sont les basfonds de collecte d'eaux usées et les lacs artificiels exposés à toutes sortes de rejets urbains. L'usage des intrants, notamment les substances agrochimiques et les fientes de poulets, est très répandu. Les dispositions réglementaires sur la distribution des produits agrochimiques ne sont pas appliquées ; ce qui favorise la circulation de pesticides appartenant à toutes les grandes familles chimiques. Les traitements chimiques ne sont pas suffisamment maîtrisés. Tous ces facteurs exposent à des risques d'intoxications et de pollutions de l'environnement. L'analyse des points d'eaux d'irrigation a mis en évidence les caractéristiques physico-chimiques très hétérogènes des eaux d'arrosage. Elle a aussi révélé une grande diversité microbienne dans ces eaux d'arrosage. Les risques sanitaires sont tout aussi diversifiés. Dans la perspective d'une meilleure protection de la santé par une réduction de la contamination de l'environnement, des actions concertées doivent être conduites, afin de mieux contrôler et réduire les risques. Cela passe par une sensibilisation de tous les acteurs, un encadrement des producteurs et une réorientation des activités avant de prendre des mesures durables comme la relocalisation des producteurs sur des sites sans risques. Une veille scientifique, mettant en œuvre des études sur la qualité physicochimique et microbiologique des eaux d'arrosage et des sols de culture, ainsi que la dynamique des producteurs doit être à mesure de fournir, constamment, des données pour aider les autorités communales à des prises de décisions efficaces.

\section{REFERENCES}

Abakou J, Brou KH. 2003. Analyse des systèmes de production et de commercialisation des cultures maraîchères dans la commune de Yamoussoukro. Mémoire pour l'obtention du Diplôme d'Ingénieurs des Techniques Agricoles, Ecole de Formation Continue et Perfectionnement des Cadres (EFCPC) de Yamoussoukro, p. 71 .

Adama E, Michel G. 2004. La filière des produits horticoles en Côte d'Ivoire. Diagnostic, objectifs et perspectives, $\mathrm{p}$. 167.

Amoah P, Drechsel P, Abaidoo RC, Ntow WJ. 2006. Pesticide and pathogen contamination of vegetables in Ghana's urban markets. Archives of Environmental Contamination and Toxicology, 50(1): 1-6.

ANADER. 2004. Rapport de présentation de l'enquête sur le développement de l'horticulture urbaine et périurbaine de Yamoussoukro, p. 21.

Bendé TJ. 2003. Production et environnement phytosanitaire des cultures légumières dans la zone urbaine de Yamoussoukro. Mémoire pour l'obtention du Diplôme d'Ingénieur des Techniques Agricoles, Ecole Supérieure d'Agronomie de Yamoussoukro. p. 63.

Delarras C, Trébaol B. 2003. Surveillance Sanitaire et Microbiologique des Eaux: Réglementation, Prélèvements, Analyses. Editions Médicales Internationales : Londres, Paris, New York. 
Diaby S. 2002. Environnement phytosanitaire des cultures maraîchères en zone urbaine et periurbaine de Yamoussoukro. Mémoire pour l'obtention du Diplôme d'Ingénieur Agronome, Ecole Supérieure d'Agronomie de Yamoussoukro, p.48.

Dümler C, Schwab A, Jäger-Mischke I, Thiam A, Stoll G, Görgen R, Prexler-Schwab S. 1993. Pesticides et Agriculture Tropicale: Dangers et Alternative. PAN-CTA.

Fondio L, Kouamé C, Djidji AH, Traoré D. 2011. Caractérisation des systèmes de culture intégrant le gombo dans le maraîchage urbain et périurbain de Bouaké dans le Centre de la Côte d'Ivoire. Int. J. Biol. Chem. Sci., 5(3): 1178-1189.

Kouablé B. 2002. Législation Phytosanitaire en Côte d'Ivoire. Ministère de l'Agriculture et des Ressources Animales : Côte d'Ivoire ; 17.

Maïga AH, Wethe J, Dembélé A, Klutse A. 2002. Valorisation des eaux usées par lagunage dans les pays en voie de développement: Etude des cas du Burkina Faso, Cameroun, Côte d'Ivoire, Ghana, Niger et Sénégal. Programme «Gestion durable des déchets et de l'assainissement urbain », Rapport final, p. 131.

N'guegang PA. 2008. L'agriculture urbaine et périurbaine à Yaoundé: analyse multifonctionnelle d'une activité montante en économie de survie. Thèse de Docteur en Sciences Agronomiques et
Ingénierie Biologique, Université Libre de Bruxelles, p. 200.

Ntow WJ. 2001. Organochlorine pesticides in water, sediment, crops, and human fluids in a farming community in Ghana. Arch. Environ. Contam. Toxicol., 40: 557-563.

Obuodi E, Keraita B, Danso G, Amoah P, Kofie OO, Rashid-Sally L, Drechsel P. 2006. Irrigated Urban Vegetable production in Ghana: Characteristics, Benefits and Risks. IWMI, RUAF and CGIAR.

Rejsek F. 2002. Analyse des eaux, Aspects réglementaires et techniques. Série Sciences et Techniques de l'Environnement.

Tellier S. 2006. Les pesticides en milieu agricole : état de la situation environnementale et initiatives prometteuses, Direction des politiques en milieu terrestre, Service des pesticides, Ministère du Développement durable, de l'Environnement et des Parcs, Gouvernement du Québec, p. 90.

Thiam A. 2007. Les pesticides sont des poisons !!! Guide des communautés pour la protection de la santé et de l'environnement. Publication de Pesticides Action Network (PAN) Africa. Série Education Environnementale $\mathrm{N}^{\circ} 2$, p. 55.

Tron I, Piquet O, Cohuet S. 2001. Effets chroniques des pesticides sur la santé : état actuel des connaissances. Observatoire Régional de Santé de la Bretagne, France : 23-90. 